\title{
CP Violation Studies in B Mesons
}

\section{On behalf of the Atlas, CDF, CMS and Do Collaborations}

\author{
Mark R. J. Williams ${ }^{1, a}$ \\ ${ }^{1}$ Indiana University, Bloomington, IN 47405, USA
}

\begin{abstract}
The four general purpose detectors on the Tevatron (CDF, D0) and LHC (Atlas, CMS) hadron colliders continue to make important measurements in the field of CP violation (CPV) in $B$ hadron physics. The CDF collaboration presents new results in direct CPV of charmless $B$ decays, providing world-leading measurements in $b$ baryon decays, and finding evidence for non zero CPV in the decay $B_{s}^{0} \rightarrow K^{-} \pi^{+}$. The D0 Collaboration presents measurements of the semileptonic mixing asymmetries in $B^{0}$ and $B_{s}^{0}$ mesons, with the former having a precision exceeding the previous world average. All four experiments perform time-dependent angular analysis of the decay $B_{s}^{0} \rightarrow J / \psi \phi$, enabling the extraction of the lifetime difference $\Delta \Gamma_{s}$ and the CPV phase $\phi_{s}^{J / \psi \phi}$. In all cases, the results obtained are consistent with each other, and with the standard model predictions.
\end{abstract}

\section{Introduction}

The violation of CP symmetry (CPV) is one of the required ingredients of baryogenesis, through which the initial matter-antimatter symmetric state produced by the big bang evolved into the matter dominated universe we observe today. In the standard model (SM), CPV in the quark sector is driven entirely by a single complex phase in the CKM quark mixing matrix. All measurements to date are consistent with this single-phase prediction [1]. However, the degree of CPV produced through this mechanism is far too small to explain matter dominance [2].

Measurements in the $B$ meson sector are particularly sensitive to any complex phases introduced in quark interactions, with several CPV observables having very small and precise predictions in the SM, but with large possible enhancements predicted by new physics models [3-6].

CPV can be broadly divided into three classes. Direct CPV occurs when a particle decay has a different rate to the corresponding charge-conjugate decay, and is quantified by the parameter

$$
A_{\mathrm{CP}}=\frac{\Gamma(\bar{B} \rightarrow \bar{f})-\Gamma(B \rightarrow f)}{\Gamma(\bar{B} \rightarrow \bar{f})+\Gamma(B \rightarrow f)} .
$$

For neutral mesons, particle-antiparticle oscillations proceed via box-diagram mediated weak interactions, and $\mathrm{CPV}$ in mixing can occur, whereby the rate of mixing $\Gamma\left(\bar{B}_{(s)}^{0} \rightarrow B_{(s)}^{0}\right)$ is different from the charge conjugate process. Finally, for a particular class of neutral meson decays, a single common final state can be reached with or without an intermediate $B$ meson oscillation. In such cases, the direct and oscillated processes interfere, and the overall complex phase driving $\mathrm{CPV}$ is the combination of the decay and mixing phases.

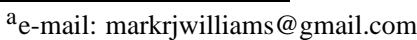

In this article, measurements of all three classes of CPV are briefly described. For full details, please see the original publications $[7,9,10,12-15]$ and references therein.

\section{Direct CP Violation in Charmless B Decays [7]}

Within the past decade, direct CP violation has been observed at greater than $5 \sigma$ significance in the charmless decay $B^{0} \rightarrow K^{+} \pi^{-}$. This unexpected result can be accommodated, although it is not strongly constrained, by the standard model, which predicts a similar effect in the corresponding charged mode $B^{+} \rightarrow K^{+} \pi^{0}$. Experimental data, however, shows a significant discrepancy between the two modes, a puzzle that has fueled much interest in these charmless decay processes. Additional high precision measurements, along with extensions into other $b$ hadron systems, can aid in future understanding of such effects, enabling SM predictions to be directly tested against new physics models. Of particular interest is the decay $B_{s}^{0} \rightarrow K^{-} \pi^{+}$, in which the SM prediction is very large, of order $30 \%$.

The CDF collaboration has performed a comprehensive study of direct CP violation in several charmless decays of $b$ hadrons, based on $9.3 \mathrm{fb}^{-1}$ of $p \bar{p}$ collisions at $\sqrt{s}=1.96 \mathrm{TeV}$, collected at the Tevatron Collider in Fermilab [7]. Four decay modes are studied, $B^{0} \rightarrow K^{+} \pi^{-}$, $B_{s}^{0} \rightarrow K^{-} \pi^{+}, \Lambda_{b}^{0} \rightarrow p \pi^{-}$, and $\Lambda_{b}^{0} \rightarrow p K^{-}$. The event sample is collected using a dedicated displaced track trigger, and subsequent offline reconstruction is optimised to select $b \rightarrow h h^{\prime}$ two-pronged decay vertices. The resulting invariant mass distribution, assuming a $\pi^{+} \pi^{-}$hypothesis for the decay products, is shown in Fig. 1. 


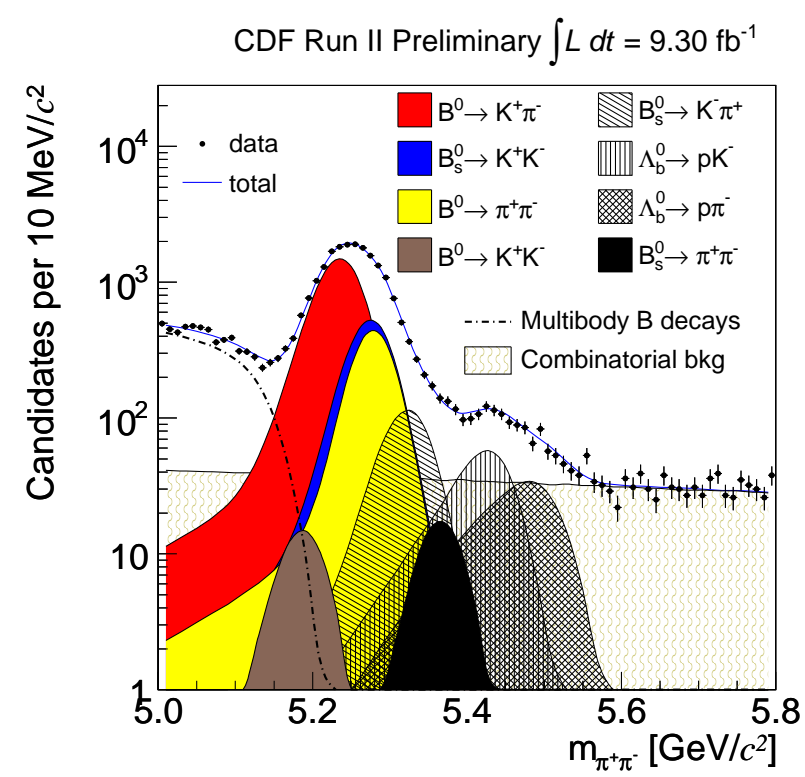

Figure 1. Invariant mass $M(\pi \pi)$ for two-pronged charmless hadronic decays, as reconstructed by the CDF experiment. Eight decays are included in the signal model, including the four with distinct final state hadrons, on which the asymmetry analysis is performed.

The yields for each process and its charge conjugate are obtained by performing a multidimensional fit to the data, including three kinematic variables: the square of the dipion mass, the scalar sum of the two final-state hadron momenta, and the momentum imbalance $\beta=\left(p^{+}-\right.$ $\left.p^{-}\right) /\left(p^{+}+p^{-}\right)$, where $p^{+(-)}$is the momentum of the positively (negatively) charged hadron. An additional 'kaonness' variable is also included to provide separation between pions and kaons in the final state.

The reconstructed yields are used to obtain the fundamental decay asymmetries, after correcting for detection asymmetries in kaon, pion and proton reconstruction, which are obtained from dedicated samples of $D^{0} \rightarrow$ $\pi^{+} K^{-}$and $\Lambda^{0} \rightarrow p \pi^{-}$decays. Production asymmetries are assumed to be zero, due to the CP-symmetric initial $p \bar{p}$ state.

For the decay $B^{0} \rightarrow K^{+} \pi^{-}$, a total of around 12000 signal events are reconstructed, and the direct CPV asymmetry is measured to be

$$
A_{C P}\left(B^{0} \rightarrow K^{+} \pi^{-}\right)=[-8.3 \pm 1.3 \pm 0.3] \%,
$$

which is consistent and competitive with the existing world average of $(-9.7 \pm 1.2) \%$. Here and elsewhere, the first quoted uncertainty is statistical, the second is systematic. In the corresponding strange decay, $B_{s}^{0} \rightarrow K^{-} \pi^{+}$, a total of around 900 signal events are used to determine

$$
A_{C P}\left(B_{s}^{0} \rightarrow K^{-} \pi^{+}\right)=[22 \pm 7 \pm 2] \%,
$$

which is consistent with the corresponding value obtained by the LHCb Collaboration, $(27 \pm 8 \pm 2) \%$ [8]. Combining the $B_{s}^{0}$ measurements from the two experiments, a combined asymmetry of $(24.2 \pm 5.4) \%$ is obtained, which is
$4.5 \sigma$ from zero. Finally, samples of around 450 and 600 events respectively are used to provide the worlds most precise measurements of the direct CPV parameter in $\Lambda_{b}^{0}$ decays:

$$
\begin{aligned}
& A_{C P}\left(\Lambda_{b}^{0} \rightarrow p \pi^{-}\right)=[7 \pm 7 \pm 3] \% \\
& A_{C P}\left(\Lambda_{b}^{0} \rightarrow p K^{-}\right)=[-9 \pm 8 \pm 4] \%,
\end{aligned}
$$

which are consistent with zero in both cases.

\section{CP Violation in Mixing [9-11]}

The presence of a non-zero complex phase in the CKM quark mixing matrix can lead to an asymmetry in the rate of neutral $B$ mesons oscillating into their antiparticles, and hence to an asymmetry in the final state decay products following oscillation. This can be quantified in terms of the semileptonic mixing asymmetry, defined as

$$
a_{\mathrm{sl}}^{q}=\frac{\Gamma\left(\bar{B}_{q}^{0} \rightarrow B_{q}^{0} \rightarrow \ell^{+} X\right)-\Gamma\left(B_{q}^{0} \rightarrow \bar{B}_{q}^{0} \rightarrow \ell^{-} X\right)}{\Gamma\left(\bar{B}_{q}^{0} \rightarrow B_{q}^{0} \rightarrow \ell^{+} X\right)+\Gamma\left(B_{q}^{0} \rightarrow \bar{B}_{q}^{0} \rightarrow \ell^{-} X\right)},
$$

where the label $q=\{d, s\}$ denotes the flavor of the light valence quark in the $B$ meson. The standard model predictions for both $a_{\mathrm{sl}}^{s}$ and $a_{\mathrm{sl}}^{d}$ are effectively negligible compared to the current experimental precision:

$$
\begin{aligned}
& a_{\mathrm{sl}}^{d}=(-0.041 \pm 0.006) \% \\
& a_{\mathrm{sl}}^{s}=(0.0019 \pm 0.0003) \% .
\end{aligned}
$$

New physics models can lead to order-of-magnitude enhancements of these asymmetries. The recent evidence for a non-zero dimuon charge asymmetry by the D0 experiment [11] can be interpreted as due to asymmetries in mixing, being sensitive to the linear combination of $B^{0}$ and $B_{s}^{0}$ mixing asymmetries. Under this interpretation, the result constrains a band in the $\left(a_{\mathrm{sl}}^{d}, a_{\mathrm{sl}}^{s}\right)$ plane which is inconsistent with the SM prediction at the 3.9 standard deviations level. As such, additional independent measurements of both $a_{\mathrm{sl}}^{s}$ and $a_{\mathrm{sl}}^{d}$ are required in order to constrain the possible sources of new physics in $B$ meson mixing and decay.

The D0 Collaboration has recently published a measurement of $a_{\mathrm{sl}}^{s}$, using a large inclusive sample of semileptonic decays $B_{s}^{0} \rightarrow \mu^{+} D_{s}^{-} X$, with $D_{s}^{-} \rightarrow \phi \pi^{-}$[9]. This analysis uses the full Tevatron Run II sample of $p \bar{p}$ collisions, corresponding to around $10.4 \mathrm{fb}^{-1}$ of integrated luminosity. Following event selection using single and dimuon triggers, and a likelihood-ratio based multivariate discriminant, a total of around 215000 signal events are reconstructed.

Events are then weighted such that the total weight in each of the four detector magnet polarity configurations is the same, a technique that causes many detector asymmetries to cancel to first order, and which is enabled by the regular reversal of both the toroidal (muon system) and solenoidal (tracking system) magnets during data collection. Weights are typically in the range $0.95-1.00$.

The raw asymmetry is defined simply in terms of the number of reconstructed events,

$$
A=\frac{N_{\mu^{+} D_{s}^{-}}-N_{\mu^{-} D_{s}^{+}}}{N_{\mu^{+} D_{s}^{-}}+N_{\mu^{-} D_{s}^{+}}} .
$$




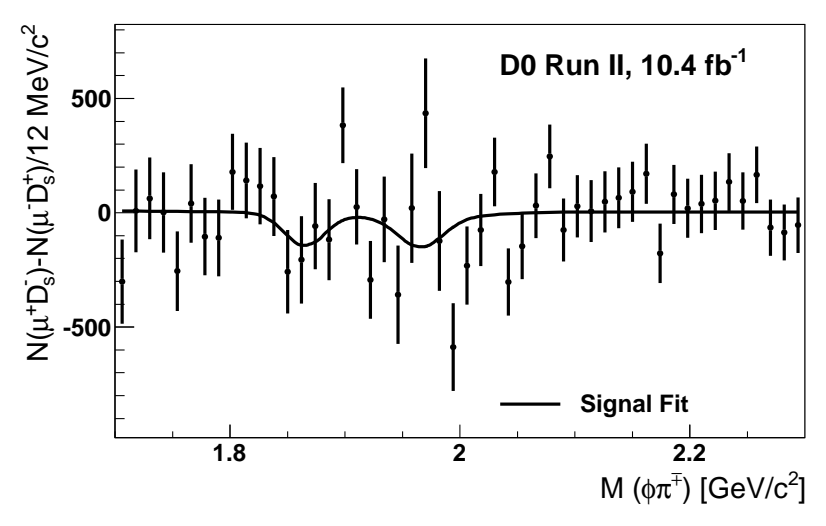

Figure 2. Difference between the $M(\phi \pi)$ distributions for positive and negative pions, used to extract the raw asymmetry of $B_{s}^{0} \rightarrow \mu^{+} D_{s}^{-} X$ events in the D0 measurement of the mixing asymmetry $a_{\mathrm{sl}}^{s}[9]$.

This is extracted using a simultaneous fit to the $M(\phi \pi)$ distribution for the sum of all events $\left[N_{\mu^{+} D_{s}^{-}}+N_{\mu^{-} D_{s}^{+}}\right]$and the difference between the charge-specific distributions $\left[N_{\mu^{+} D_{s}^{-}}-N_{\mu^{-} D_{s}^{+}}\right]$. The difference distribution is shown in Fig. 2; the consistency with zero asymmetry outside of the signal region demonstrates that the residual reconstruction asymmetries are small. Charge-randomised ensemble tests confirm that the fit is unbiased and returns the appropriate statistical uncertainty on the raw asymmetry.

The raw asymmetry is related to the underlying physical asymmetry via the relation

$$
a_{\mathrm{sl}}^{s}=\frac{A-A_{\mathrm{BG}}}{F_{B_{s}^{0}}^{\mathrm{osc}}} .
$$

Here, $A_{\mathrm{BG}}$ is the residual detector asymmetry remaining for reconstructing the final state particles $\mu^{ \pm} \phi(\rightarrow$ $\left.K^{+} K^{-}\right) \pi^{\mp}$, and $F_{B_{s}^{0}}^{\text {osc }}$ is the fraction of reconstructed $\mu D_{s}^{0}$ candidates that originate from $B_{s}^{0}$ mesons which have oscillated into their antiparticle prior to decay. This denominator is a dilution factor that accounts for other charge symmetric sources of $\mu D_{s}^{0}$ events, such as $B^{+}$decay, direct decays of $B_{s}^{0}$ mesons, and prompt production of charm hadrons incorrectly associated with a reconstructed muon.

For this decay, the total residual detector asymmetry is very small, $A_{\mathrm{BG}}=(0.13 \pm 0.06) \%$. The largest component is from muon reconstruction, at $(0.11 \pm 0.03) \%$, which is measured using $J / \psi \rightarrow \mu^{+} \mu^{-}$decays using a tag and probe method. The pion reconstruction asymmetry is determined to be negligible, using dedicated samples of $K_{S}^{0} \rightarrow \pi^{+} \pi^{-}$and $K^{* \pm} \rightarrow K_{S}^{0} \pi^{ \pm}$decays, and is assigned a systematic uncertainty of $\pm 0.05 \%$. There is a small additional asymmetry in the reconstruction of $\phi \rightarrow K^{+} K^{-}$due to the underlying kaon asymmetry, in conjunction with the asymmetric kinematics of this decay. The kaon asymmetry is measured in $K^{* 0} \rightarrow K^{+} \pi^{-}$decays, leading to an asymmetry $A_{\phi}=(0.020 \pm 0.002) \%$.

The dilution factor $F_{B_{s}^{0}}^{\mathrm{osc}}=0.465 \pm 0.017$ is determined using Monte Carlo simulation. Around $93 \%$ of all $\mu D_{s}^{0}$ candidates are found to originate from $B_{s}^{0}$ decay; the ap- propriate mixing relation is then used to determine the fraction of these mesons that have oscillated into their antiparticle prior to decay, a number which is very close to $50 \%$ due to the high mixing frequency $\Delta M_{s}$ with respect to the particle lifetime.

Combining these measurements using Eq. (10), the semileptonic mixing asymmetry in $B_{s}^{0}$ mesons is measured to be

$$
a_{\mathrm{sl}}^{s}=(-1.12 \pm 0.74 \pm 0.17) \% .
$$

This is consistent with both the SM prediction and with the contours obtained from the D0 dimuon asymmetry analysis.

The D0 Collaboration has also performed the equivelent analysis on $B^{0}$ mesons, using the same dataset, in decays $B^{0} \rightarrow \mu^{+} D^{(*)^{-}} X[10]$. Two independent decay channels are used to reconstruct the charm mesons, $D^{*-} \rightarrow$ $\bar{D}^{0} \pi^{-}, \bar{D}^{0} \rightarrow K^{+} \pi^{-}(\sim 520000$ signal events after magnet weighting), and $D^{-} \rightarrow K^{+} \pi^{-} \pi^{-}$( 720000 signal events after magnet weighting). The analyses are performed separately, with the final measurements of $a_{\mathrm{sl}}^{d}$ being combined.

The overall analysis strategy is the same as for the measurement of $a_{\mathrm{sl}}^{s}$, although there are two principal differences in the application of this method. First, the small value of $\Delta M_{d}$ with respect to the $B^{0}$ lifetime results in a strong dependence of the dilution fraction $F_{B_{d}^{0}}^{\text {osc }}$ on the reconstructed decay length. Candidates with a short decay length are most likely to originate from $B^{0}$ mesons which have not oscillated, or from prompt production of charm hadrons, while candidates at large decay lengths are most likely to originate from the decay of oscillated $B^{0}$ mesons. To exploit this behaviour, the analysis is performed in six bins of visible proper decay length (VPDL), with the first two bins used as a control region in which the observed raw asymmetry should be dominated by detector effects.

Second, the presence of a single charged kaon in the final state significantly increases the reconstruction asymmetries that must be subtracted in order to obtain the underlying mixing asymmetry. This is because $K^{+}$mesons have a significantly longer average path length than $K^{-}$ mesons while traversing the detector material (due to the absense of a positive analogue of $K^{-} p \rightarrow \Lambda^{0} n$ or other hyperon-producing interactions), and hence are more likely to be reconstructed as charged tracks.

Figure 3 shows an example of the difference fit for the most sensitive VPDL bin in the $\mu D^{*}$ channel. The significant raw asymmetry is expected as a result of the kaon reconstruction asymmetry, and can be compared with Fig. 2 in which there is no single charged kaon in the final state. The overall background asymmetry correction $A_{\mathrm{BG}}$ is dominated by this kaon effect, and typically lies in the range $1.18-1.26 \%$, depending on the channel and VPDL region. In addition to the kaon asymmetry, the muon and pion reconstruction asymmetries are also taken into account (in the latter case, just via an additional source of systematic uncertainty).

The dilution factor $F_{B_{d}^{0}}^{\text {osc }}$ is determined to be around 0.01-0.02 for the control region, rising to around 0.70 for 


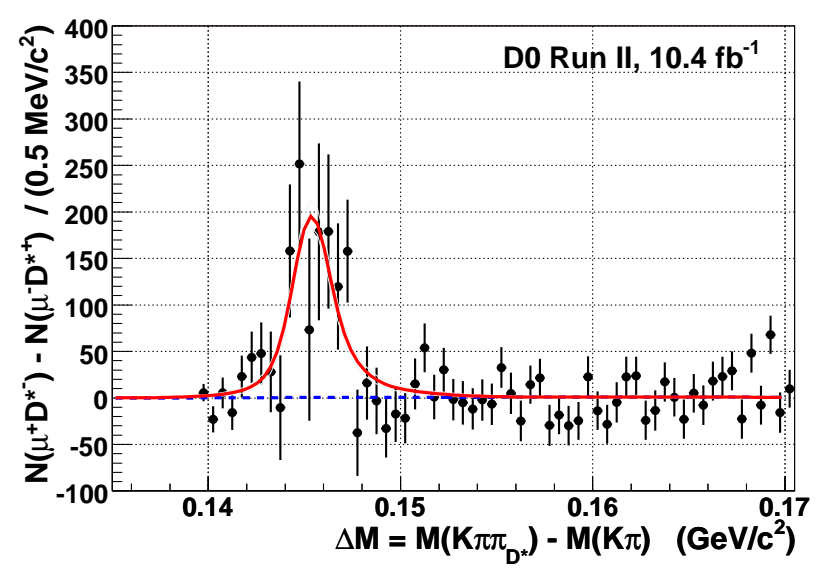

Figure 3. Difference between the invariant mass distributions $\Delta M=M\left(D^{0} \pi^{ \pm}\right)-M\left(D^{0}\right)$ for positive and negative pions, used to extract the raw asymmetry of $B^{0} \rightarrow \mu^{+} D^{*-} X$ events in the D0 measurement of the mixing asymmetry $a_{\mathrm{sl}}^{d}$ [9]. The significant peak is expected due to the asymmetry in charged kaon reconstruction.

the largest VPDL bin. This takes into account the possible effect of the $B_{s}^{0}$ mixing asymmetry, although contamination from $B_{s}^{0}$ decays is small, of order $1-3 \%$. The contribution from $B^{+}$decays is the largest background, building from around $5-15 \%$ as the VPDL increases.

Eight individual measurements of $a_{\mathrm{sl}}^{d}$ are made, comprising four VPDL regions for each of the two channels. These are then combined into two channel-specific measurements, and finally into a single overall result, with all correlations properly taken into account in the combination:

$$
\begin{aligned}
a_{\mathrm{sl}}^{d}(\mu D) & =[0.43 \pm 0.63 \pm 0.16] \%, \\
a_{\mathrm{sl}}^{d}\left(\mu D^{*}\right) & =[0.92 \pm 0.62 \pm 0.16] \%, \\
a_{\mathrm{sl}}^{d}(\text { combined }) & =[0.68 \pm 0.45 \pm 0.14] \% .
\end{aligned}
$$

This result is the most precise to date, with smaller uncertainties than the existing world average from $B$ factories. The D0 results for $a_{\mathrm{sl}}^{s}$ and $a_{\mathrm{sl}}^{d}$ can be combined with the constraints from the dimuon asymmetry [11], and the existing $a_{\mathrm{sl}}^{d}$ measurements from $B$ factories, in a twodimensional fit to obtain the following values:

$$
\begin{aligned}
& a_{\mathrm{sl}}^{d}(\text { comb. })=(0.07 \pm 0.27) \%, \\
& a_{\mathrm{sl}}^{s}(\text { comb. })=(-1.67 \pm 0.54) \%,
\end{aligned}
$$

where the two parameters have a correlation coefficient of -0.46 . The fit returns a $\chi^{2}$ of 2.0 for 2 degrees-of-freedom. The $p$-value of the combination with respect to the SM point is 0.0037 , corresponding to an inconsistency at the 2.9 standard deviation level. The contours are shown in Fig. 4.

\section{CP Violation in Interference [12-15]}

For the process $B_{s}^{0} \rightarrow J / \psi \phi$, the direct decay and the decay following mixing $B_{s}^{0} \rightarrow \bar{B}_{s}^{0} \rightarrow J / \psi \phi$ interfere, giving sensitivity to the relative phase between the mixing and decay

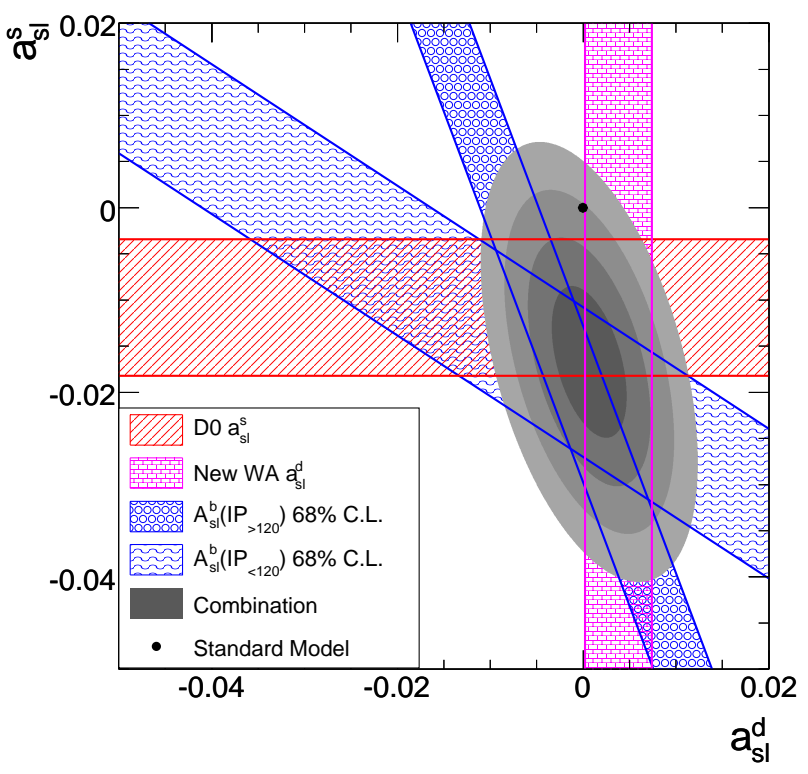

Figure 4. Combination of measurements of $a_{\mathrm{sl}}^{d}$ (D0 [10] and existing world-average from B factories [1]), $a_{\mathrm{sl}}^{s}$ (D0 [9]), and the two impact-parameter-binned constraints from the samecharge dimuon asymmetry $A_{\mathrm{sl}}^{b}$ (D0 [11]). The bands represent the \pm 1 standard deviation uncertainties on each measurement. The ellipses represent the 1,2,3, and 4 standard deviation twodimensional confidence level regions of the combination.

amplitudes, $\phi_{s}^{J / \psi \phi} \equiv-2 \beta_{s}=-\arg \left[-V_{t b} V_{t s}^{*} / V_{c b} V_{c s}^{*}\right]$. The $\mathrm{SM}$ predicts a value $\phi_{s}^{J / \psi \phi}=-0.04 \pm 0.01$, whereas new physics models can enhance this phase significantly.

Experimentally, the extraction of this phase presents several challenges. The vector-vector final state can take three possible angular momentum configurations, and hence three polarizations, two CP-even $(L=0,2)$ and one CP-odd $(L=1)$. In addition, there can be a contribution from $J / \psi K^{+} K^{-}$, with non-resonant S-wave $K^{+} K^{-}$. The total decay amplitude hence comprises four distinct components, each of which exhibits a time dependence that is a function of the underlying physical parameters $\left(\tau_{s}, \Delta M_{s}, \Delta \Gamma_{s}, \phi_{s}^{J / \psi \phi}\right)$.

The decay width then contains ten terms, of which six are due to interference, and an angular analysis must be performed in order to disentangle these components. Each of the analyses presented here chooses the transversity basis to define the three angles, and uses simultation to determine the detector angular acceptance and efficiency. The physics parameters are extracted using a multidimensional fit over the measured decay time, three angles, invariant mass, and often additional variables which help to constrain experimental effects (such as the decay time resolution, or mass resolution).

Without knowledge of the initial flavor $\left(B_{s}^{0}\right.$ or $\left.\bar{B}_{s}^{0}\right)$ at production, the measured decay width is the average of the two relations, which introduces a twofold ambiguity into the measurement of $\phi_{s}^{J / \psi \phi}$. A flavor-tagged analysis helps to resolve this ambiguity, but adds additional complexity 
to the analysis and requires careful study to understand the efficiency and mis-tag rate of the method.

The CMS Collaboration has performed an untagged angular analysis of this decay, using $5 \mathrm{fb}^{-1}$ of $p p$ collisions at $\sqrt{s}=7 \mathrm{TeV}$ [12], resulting in around 15000 reconstructed signal candidates. As a first step towards a full measurement of the phase, they extract other parameters under the assumption $\phi_{s}^{J / \psi \phi}=0$; with this condition, the $B_{s}^{0}$ mass eigenstates are also well-defined CP-eigenstates. The lifetime and lifetime difference for heavy and light states are respectively measured to be

$$
\begin{aligned}
c \tau_{s} & =[458.0 \pm 5.9 \pm 2.2] \mu \mathrm{m}, \\
\Delta \Gamma_{s} & =[0.048 \pm 0.024 \pm 0.003] \mathrm{ps}^{-1}
\end{aligned}
$$

where the precision is constrained by the limited size of the data sample.

The Atlas Collaboration has also performed an untagged angular analysis, using $4.9 \mathrm{fb}^{-1}$ of $p p$ collisions at $\sqrt{s}=7 \mathrm{TeV}$ [13]. A total of around 23000 signal candidates are reconstructed. The complex phase is measured directly, along with other parameters. To resolve the ambiguities arising from the untagged method, external constraints are applied: the strong phase $\delta_{\perp}$ is constrained to $(2.95 \pm 0.39)$ as obtained by the LHCb Collaboration [16], and the solution with negative $\Delta \Gamma_{s}$ is rejected. The physics parameters are measured to be

$$
\begin{aligned}
\phi_{s}^{J / \psi \phi} & =[0.22 \pm 0.41 \pm 0.10] \mathrm{rad} \\
\Delta \Gamma_{s} & =[0.053 \pm 0.021 \pm 0.008] \mathrm{ps}^{-1} \\
\Gamma_{s} & =[0.677 \pm 0.007 \pm 0.004] \mathrm{ps}^{-1}
\end{aligned}
$$

The phase is in good agreement with the SM prediction. The two-dimensional contours in $\left(\phi_{s}^{J / \psi \phi}, \Delta \Gamma_{s}\right)$ are shown in Fig. 5.

Both the D0 and CDF Collaborations have performed the full flavor-tagged analysis of this decay mode. Recently the CDF collaboration published an update using the full Tevatron Run II sample $\left(9.6 \mathrm{fb}^{-1}\right)$ of $p \bar{p}$ collisions at $\sqrt{s}=1.96 \mathrm{TeV}$ [14]. The flavor of the $B_{s}^{0}$ meson at production is determined on a statistical basis using both 'same-side' (only used for the first $5.2 \mathrm{fb}^{-1}$, with a tagging power of $3.5 \pm 1.4 \%$ ) and 'opposite-side' (used for the full sample, with a tagging power of $1.39 \pm 0.05 \%$ ) taggers. A total of around 11000 signal events are reconstructed, and the fit uses no external constraints other than the wellknown mixing frequency $\Delta M_{s}=17.77 \pm 0.12 \mathrm{ps}^{-1}$ as previously measured by $\mathrm{CDF}$.

Point estimates for several parameters can be extracted directly from the fit, if $\phi_{s}^{J / \psi \phi}$ is fixed to zero. These include the lifetime and lifetime difference

$$
\begin{aligned}
\tau_{s} & =[1.528 \pm 0.019 \pm 0.009] \mathrm{ps} \\
\Delta \Gamma_{s} & =[0.068 \pm 0.026 \pm 0.009] \mathrm{ps}^{-1}
\end{aligned}
$$

Confidence regions in $\left(\phi_{s}^{J / \psi \phi}, \Delta \Gamma_{s}\right)$ are extracted using a profile-likelihood ratio statistic, which properly takes into account the effect of correlations, biases from the fit, and systematic uncertainties. Statistical ensemble tests are used to estimate the effect of 30 nuisance parameters, which inflates the confidence regions by around $40 \%$.
The final confidence region at the $68 \%$ C.L. are shown in Fig. 5, in which an equivalent solution with negative $\Delta \Gamma_{s}$ has been removed. The remaining region is consistent with the SM prediction.

\section{Summary}

The general purpose detectors at the Tevatron and LHC colliders have demonstrated the ability to perform precise measurements of CP-violation parameters, using innovative techniques. The CDF Collaboration has performed an analysis of several charmless decays of $B$ hadrons, providing world-leading measurements of direct CPV parameters, and confirming the $\mathrm{LHCb}$ evidence for non-zero CPV in $B_{s}^{0} \rightarrow K^{-} \pi^{+}$decays [7]. The D0 Collaboration has measured the semileptonic mixing asymmetries in both $B^{0}$ and $B_{s}^{0}$ decays with world-leading precision $[9,10]$. The results agree with both the SM predictions, and the existing measurement of the same-charge dimuon asymmetry [11].

All four experiments have performed time-dependent angular analysis of the decay $B_{s}^{0} \rightarrow J / \psi \phi[12-15]$, with all results in agreement with the standard model predictions of both the lifetime difference $\Delta \Gamma_{s}$ and the CP-violating phase $\phi_{s}^{J / \psi \phi}$. With the rapidly increasing dataset of Atlas and CMS, future measurements of these parameters should have significantly better precision and rely less on external inputs.

\section{References}

[1] J. Beringer et al. (Particle Data Group), Phys. Rev. D 86, 010001 (2012), and http://www.slac.stanford.edu/ xorg/hfag/osc/spring_2012/.

[2] P. Huet and E. Sather, Phys. Rev. D 51, 379 (1995)

[3] M. S. Carena, J. M. Moreno, M. Quiros, M. Seco and C. E. M. Wagner, Nucl. Phys. B 599, 158 (2001).

[4] W. S. Hou, Chin. J. Phys. 47, 134 (2009).

[5] S. Tulin and P. Winslow, Phys. Rev. D 84, 034013 (2011).

[6] J. M. Cline, K. Kainulainen and M. Trott, J. High Energy Phys. 11, 089 (2011).

[7] T. Aaltonen et al. (CDF Collaboration), Phys. Rev. Lett. 106, 181802 (2011), and update in CDF Public Note 10726.

[8] R. Aaij et al. [LHCb Collaboration], Phys. Rev. Lett. 108, 201601 (2012) [arXiv:1202.6251 [hep-ex]].

[9] V. M. Abazov et al. [D0 Collaboration], Phys. Rev. Lett. 110, 011801 (2013)

[10] V. M. Abazov et al. [D0 Collaboration], Phys. Rev. D 86, 072009 (2012)

[11] V. M. Abazov et al. (D0 Collaboration), Phys. Rev. D 84, 052007 (2011).

[12] CMS Collaboration, CMS-PAS-BPH-11-006.

[13] G. Aad et al. [ATLAS Collaboration], arXiv:1208.0572 [hep-ex].

[14] T. Aaltonen et al. [CDF Collaboration], Phys. Rev. Lett. 109 (2012) 171802 


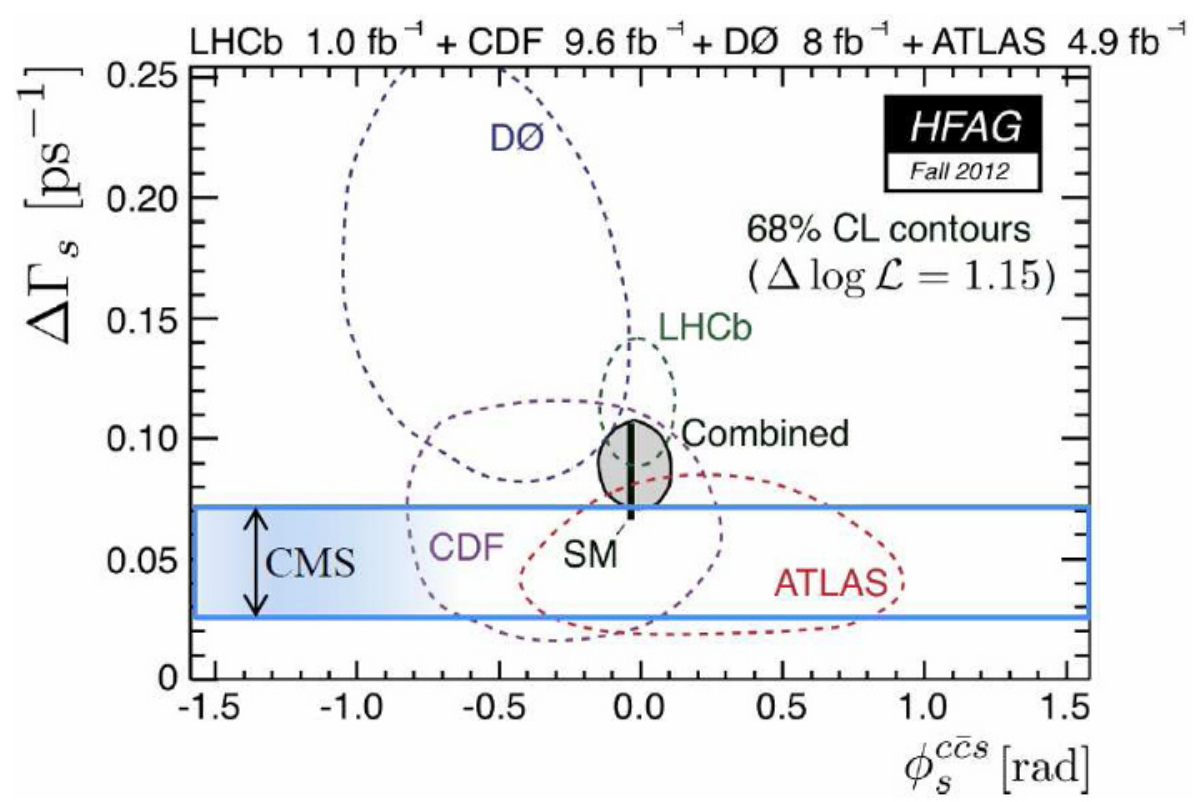

Figure 5. Summary of measurements [1] of $\left(\phi_{s}^{J / \psi \phi}, \Delta \Gamma_{s}\right)$ from the decay $B_{s}^{0} \rightarrow J / \psi \phi$, from the Atlas [13], CDF [14], D0 [15] and $\mathrm{LHCb}[16]$ collaborations. Also shown is the corresponding measurement of the lifetime difference from the CMS Collaboration [12].

[15] V. M. Abazov et al. [D0 Collaboration], Phys. Rev. D 85, $032006(2012)$
[16] R. Aaij et al. [LHCb Collaboration], Phys. Rev. Lett. 108, 101803 (2012) 